\title{
Using the Assessment for Signal Clients as a Feedback Tool for Reducing Treatment Failure
}

\author{
Melissa Mallory White \\ Brigham Young University
}

Follow this and additional works at: https://scholarsarchive.byu.edu/etd

Part of the Psychology Commons

\section{BYU ScholarsArchive Citation}

White, Melissa Mallory, "Using the Assessment for Signal Clients as a Feedback Tool for Reducing Treatment Failure" (2016). Theses and Dissertations. 6092.

https://scholarsarchive.byu.edu/etd/6092

This Thesis is brought to you for free and open access by BYU ScholarsArchive. It has been accepted for inclusion in Theses and Dissertations by an authorized administrator of BYU ScholarsArchive. For more information, please contact scholarsarchive@byu.edu, ellen_amatangelo@byu.edu. 
Using the Assessment for Signal Clients as

a Feedback Tool for Reducing

Treatment Failure

Melissa Mallory White

A thesis submitted to the faculty of

Brigham Young University

in partial fulfillment of the requirements for the degree of

Master of Science

Michael J. Lambert, Chair

Bruce N. Carpenter

Benjamin M. Ogles

Department of Psychology

Brigham Young University

July 2016

Copyright (C) 2016 Melissa Mallory White

All Rights Reserved 


\author{
ABSTRACT \\ Using the Assessment for Signal Clients as \\ a Feedback Tool for Reducing \\ Treatment Failure \\ Melissa Mallory White \\ Department of Psychology, BYU \\ Master of Science
}

The Clinical Support Tools (CST) was developed to help therapists organize and target potential problems that might account for negative outcomes in psychotherapy. The core of CST feedback is The Assessment for Signal Clients (ASC). The purpose of this study was to describe and identify patterns of problems that typically characterize off-track cases. A cluster analysis of 107 off-track clients revealed three client types: those whose problems were characterized by alliance and motivational difficulties; those characterized by social support and life event difficulties; and those whose problems had an indistinguishable pattern. Loglinear modeling showed that if patients had less therapeutic alliance problems they were also less likely to have motivational problems. Findings were also consistent with the cluster analysis, which showed that a relatively higher percentage of not-on-track participants received signal alerts for the social support items and scale. Individuals whose progress goes off-track appear to have their greatest difficulty with social support, losses, and therapy task agreement.

Keywords: treatment failure, progress feedback, psychotherapy outcome, therapeutic alliance 


\section{ACKNOWLEDGEMENTS}

I would like to express my gratitude to my parents, John and Dianna White, and my grandmother, Janice A. Corallo. Thank you for your continued belief and encouragement while I've pursued my dreams, especially during the times I've lacked the belief in myself. Thank you for picking up the phone to talk and for taking me all over the country in the hopes of teaching me what hard work and dedication is. Your sacrifice has taught me those lessons.

I would also like to thank Dr. Michael J. Lambert, Dr. Ben Ogles, Dr. Russ Bailey and Stevie Mclaughlin for working with me on this project. It never would have come together without your help. Specifically, I would like to thank Dr. Lambert for his continued support throughout my professional development. 


\section{TABLE OF CONTENTS}

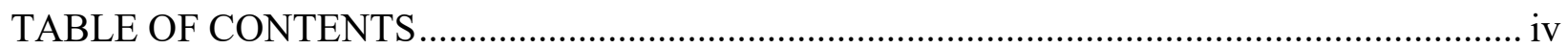

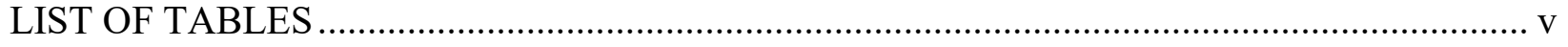

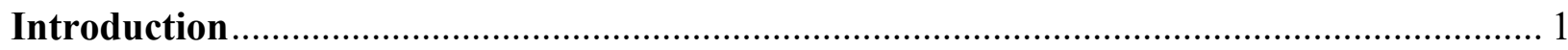

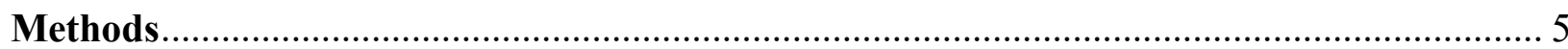

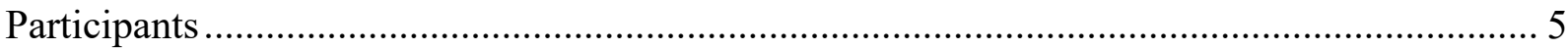

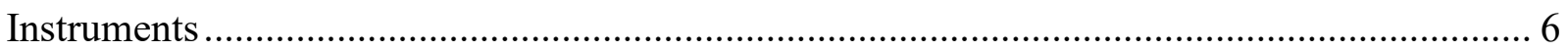

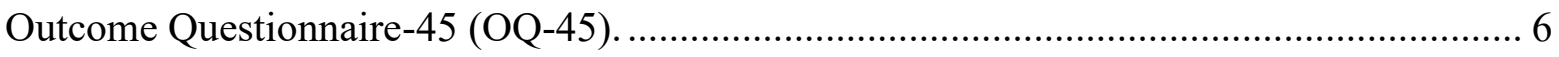

Assessment for Signal Clients (ASC) ................................................................................ 7

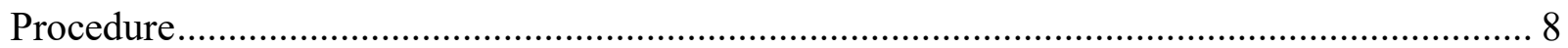

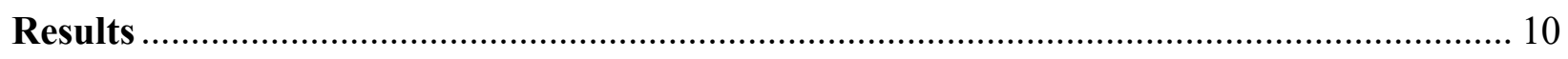

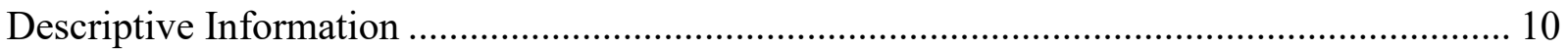

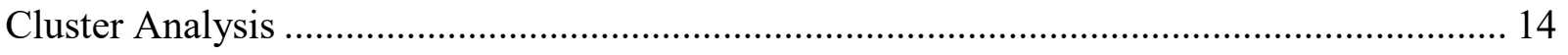

Loglinear Modeling: Examination of Associations between Variables................................... 17

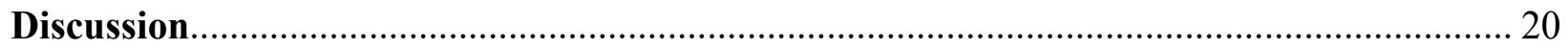

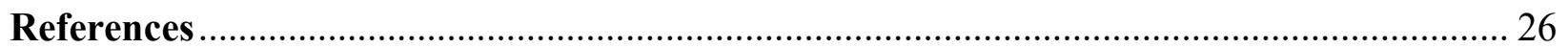




\section{LIST OF TABLES}

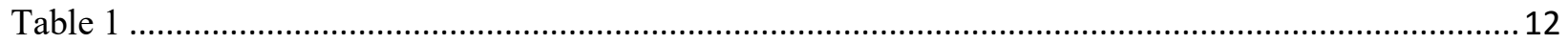

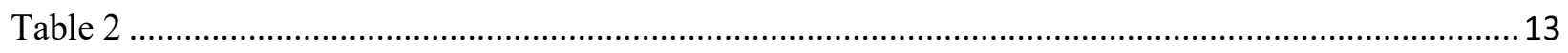

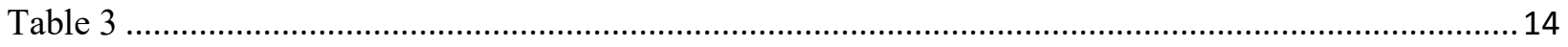

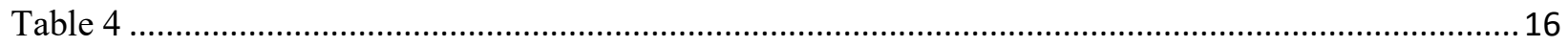

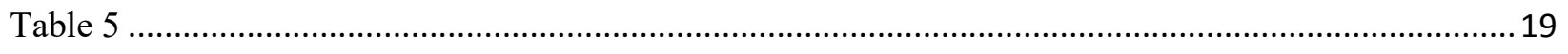

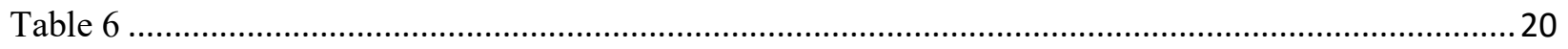




\section{Using the Assessment for Signal Clients as \\ a Feedback Tool for Reducing \\ Treatment Failure}

\section{Introduction}

Lambert and colleagues at Brigham Young University have developed methods of predicting and preventing deterioration in psychotherapy by alerting clinicians that a client is predicted to leave treatment in a deteriorated state. As part of this effort they developed Clinical Support Tools (CST; Lambert, Bailey, Kimball, Shimokawa, Harmon, \& Slade, 2007) to help therapists organize and target potential problems that might account for negative outcomes. Shimokawa, Lambert, and Smart (2010) performed a mega-analysis of clinical trials carried out by the BYU group and found a large effect size between no feedback clients (treatment-as-usual) and progress feedback clients plus Clinical Support Tools $(d=.70)$ treated by the same therapists. The core of Clinical Support Tool feedback is a 40-item self-report questionnaire (Assessment for Signal Clients; ASC) that is given to those clients who are predicted to deteriorate. The Assessment for Signal Clients attempts to identify problems with the therapeutic alliance, motivation, social support, and problematic life events. ASC feedback provides clinicians with information regarding which areas and which specific items are significantly below normative values. The ASC feedback thereby alerts therapists to possible target areas for problem-solving in therapy. The Assessment for Signal Clients results are provided to therapists by the OQ-Analyst--software that also provides recommendations for interventions that therapists can consider using.

The Assessment for Signal Clients targets general areas considered important across types of psychotherapy and a wide range of disorders common in adult-treatment, outpatient practice. The Clinical Support Tools also provide a decision tree to organize therapist problem- 
solving, directing therapists to first consider the strength of the therapeutic relationship, then motivation, then social supports external to psychotherapy, followed by negative life events, all of which are measured by the Assessment for Signal Clients. The decision tree then directs therapists to reconsider the patient's diagnosis and the possible need for medication referral and reconsideration of the treatment method. While the Clinical Support Tool feedback cannot provide prescriptive information for getting the patient back on track for a positive outcome, clinicians have found the Clinical Support Tool feedback useful in their work with clients, with experimental data demonstrating reduced deterioration rates in predicted treatment failures from a baseline of $20 \%$ to $5.5 \%$ and the rate of positive outcomes from $22 \%$ to $50.5 \%$ (Shimokawa et al., 2010). We cannot attribute the success of the Clinical Support Tool intervention to the use of the Assessment for Signal Clients but hypothesize it is essential because of its focus on specific areas of client functioning.

In creating the Assessment for Signal Clients we considered its primary function to be quantification of the quality of the psychotherapy relationship given the strength of the empirical relationship between client's perception of therapist attitudes and behavior and the outcome of psychotherapy (Horvath, Del Re, Flückiger, \& Symonds, 2011). The relationship may be of special importance in negative treatment as alliance ruptures occur over the course of therapy and can be repaired by the therapist (Safran \& Muran, 2000). Given that the therapist's perception of their relationship with the client does not have as strong a relationship with the client perception of the relationship, there is a specific need to provide therapists with clientreported perceptions, including specific indicators of alliance problems. This is facilitated through the use of cut scores that delineate a "problem" or "no problem" with particular aspects of the relationship. In the case of the Assessment for Signal Clients, the therapeutic alliance was operationalized with the possibility that the alliance, in general, was problematic but also 
assumed that any of the alliance could be problematic or not. Since the alliance is made up of the strength of the affective bond, agreement on therapeutic tasks and agreement of goals, cut-off scores can be provided for each. In addition, while a specific aspect (bond, task or goal) may not be problematic, individual items within each area could be. If a risk of a negative outcome is detected during therapy, evaluating specific items within the alliance that are below normative values could speed problem-solving and lead to corrective actions.

Another important variable that is included in the Assessment for Signal Clients and that has been shown to affect outcome is client motivation (Bohart \& Wade, 2013). Specifically, clients who worsen during treatment may lose or lack motivation for psychotherapy because they fail to have their expectations met or become discouraged with their progress. The extent to which a client is motivated to change while in therapy varies among clients. According to the stages of change model, clients move through motivational stages throughout treatment (Prochaska \& Norcross, 2003). This model of change shows that people who are in the process of changing a behavior will cycle through low levels of motivation to high levels of motivation to change. This means that a lack of success in therapy could be associated with a client's motivation and the degree to which therapists' attitudes and behaviors are responsive to motivational stages. Given this information, when creating the Assessment for Signal Clients it was hypothesized that it would be important for the therapist to become aware of motivational problems and respond to them if the client went off-track.

Two "outside of therapy" variables that were also included in the Assessment for Signal Clients are social support and life events. Social support is the degree to which clients feel their family and friends support them, i.e., that the client can rely on a support system. Social support can be defined through the amount of interpersonal connection an individual has with others and the quality of those relationships (Chronister, Johnson, \& Berven, 2006). Research suggests that 
strong interpersonal relationships reduce psychological disturbance and augment the effectiveness of therapy (Zimet, Dahlem, Zimet, \& Farley, 1988; Thoits, 1986). In addition, having adequate social support seems to help with long term benefits of psychotherapy. For example, Lindfors, Ojanen, Jääskeläinen, \& Knekt (2014) found at a three-year follow-up that patients with a low level of social support before treatment did not benefit as much from psychotherapy as those with high levels of support. Furthermore, when a client is predicted to deteriorate in therapy, they have lower amounts of social support compared to those who are progressing in therapy (Probst, Lambert, Loew, Dahlbender, \& Tritt, 2014a). Likewise, situations outside of therapists control may contribute to deterioration, such as negative life events (Lambert \& Cattani-Thompson, 1996). Life events characterized by loss as well as physical pain appear to be of particular importance and may need to be addressed in therapy (Lambert et al., 2007).

Although there is evidence showing that the use of OQ alarm signals plus Clinical Support Tools feedback reduces deterioration in off-track clients and substantially bolsters positive outcomes, little is known about the frequency with which off-track clients subscribe to specific items and scales within the Assessment for Signal Clients. Details about the factors associated with flagging progress in therapy, particularly from the client's perspective, may provide rich clinical material to psychotherapists and possibly answer specific questions about deteriorating clients. The information gathered may shed light on which items and scales (alliance, motivation, social support, life events) are most often problematic in off-track cases. In addition, identifying patterns of problems across off-track clients (i.e., clustering patients into typical types) and understanding relationships between the Assessment for Signal Clients four problems may be determined. Such research has yet to be undertaken and more empirical data 
could help clinicians use the Assessment for Signal Clients to enhance their problem-solving in therapy for those clients predicted to leave treatment in a deteriorated state.

\section{Methods}

\section{Participants}

Participant data was drawn from a previously published study carried out in a hospital-based outpatient clinic in the Western United States (Simon, Lambert, Harris, Busath, \& Vazquez, 2012). Of the 464 patients who consented to participate in that study and were randomly assigned to either a feedback condition or a treatment as usual condition, 107 completed the Assessment for Signal Clients (ASC) after they were identified as not-on-track (NOT) to have a positive treatment outcome. Completion of the ASC took place immediately after they first signaled as being off-track. The session at which the patient signaled varied from patient to patient.

Prior to completion of the of the Simon et al. (2012) study, the participants' data were deidentified. The de-identified data as well as the descriptive information for the overall sample was available to the researchers of this study. For the whole sample the mean age was 36.10 years $(S D=13.32)$. Sixty-four percent of the sample were female with $93 \%$ being Caucasian. The primary diagnosis for the participants was mood disorders (64\%), followed by anxiety disorders $(30 \%)$ and substance abuse $(5 \%)$ with $46 \%$ meeting criteria for two or more disorders. Fifty-nine percent of participants were married and 59\% were currently employed. Fourteen percent of participants had received individual psychotherapy in the past and $74 \%$ were taking psychoactive medication when they entered individual psychotherapy at the beginning of treatment. 


\section{Instruments}

Outcome Questionnaire-45 (OQ-45). Client level of disturbance and identification as an off-track case was based on the Outcome Questionnaire (OQ-45; Lambert et al., 2013), and algorithms for identifying off track cases were embedded in the OQ-Analyst software. The OQ45 was designed to measure client progress in therapy and to be repeatedly administered during the course of treatment and at termination. It assesses three aspects of the client's life: subjective discomfort/symptoms; problems in interpersonal relationships; and problems in social role performance. The items also measure personally and socially relevant characteristics that affect the individual's quality of life, attempting to quantify both positive and negative functioning. Each item is scored on a 5-point scale and the total score yields a range of possible scores of 0 to 180, where higher values indicate higher levels of client distress and pathology.

Completion of the OQ-45 takes approximately five to seven minutes and is typically administered prior to each treatment session. When clients complete the measure they are instructed to reflect back over the last week. The OQ-45 has adequate internal consistency $(r=$ $.93)$ and three-week test-retest reliability $(r=.84)$. Concurrent validity is moderate to high $(r$ $=.50-.85)$ when correlated with measures most often used to assess psychotherapy outcome in clinical trials such as the Symptom Checklist-90 and Beck Depression Inventory. Most importantly, the OQ-45 has been shown to be sensitive to change in clients over short time periods while remaining stable in untreated individuals (Vermeersch, Lambert, \& Burlingame, 2000; Vermeersch, et al., 2004).

Further details about the OQ-45 can be found in the Administration and Scoring Manual (Lambert, et al., 2013). Most relevant to the current study is the accuracy identifying treatment failures. Finch, Lambert, and Schaajle (2001) developed algorithms to identify cases at risk for 
deterioration based on a sample of more than 11,000 patients receiving treatment from across the USA. The actuarial method developed for the OQ-45 has been tested in four studies (Ellsworth, Lambert, \& Johnson, 2006; Hannan, et al., 2005; Lambert et al., 2002; Spielmans, Masters, \& Lambert, 2006) and they appear to be successful at predicting which patients will have negative treatment outcomes, accurately identifying from 80 to $100 \%$ of clients who are deteriorated at the end of therapy.

Assessment for Signal Clients (ASC). The ASC is a 40-item self-report scale that enquires into patient experiences over the prior week using a five-point Likert scale with anchors ranging from strongly agree to strongly disagree. It has four scales: Therapeutic Alliance (11 items), Motivation for Therapy (9 items), Social Support (11 items), and Life Events (9 items) but a total score is not calculated.

Therapeutic Alliance items inquire about the therapeutic bond, shared goals, and agreement on therapeutic tasks, as well as alliance rupture. Motivation items focus on low or inadequate motivation for treatment, including negative reactions to the treatment process and negative expectations. Social support items inquire into the degree to which individuals feel that their family and close friends can be counted on. Life event items focus on recent negative life events related to loss and illness and are intended to alert therapists to life crises that might need to be addressed in therapy.

The Assessment for Signal Clients feedback report is given to the therapist and consists of a scale score for each aspect of the alliance, along with a cut-off score signaling if there is an overall problem in any of the four scales. Identification of a problematic scale or item score was based on normative data. The normative data included 400 individuals participating in psychotherapy. One hundred and sixty-nine of these individuals were served in a community 
mental health setting that included lower socioeconomic status and severe psychopathology with the rest coming from a university counseling center setting. For an item (or scale) to be potentially problematic it had to be subscribed to by $20 \%$ or fewer of the normative sample. The rationale for providing individual item feedback is that it enhances clinician problem-solving by making feedback more specific. From the specific answers to these questions, a report is given indicating to the therapist which scale questions may be a possible concern. Of note, according to the present state of the literature, there is no evidence supporting the use of specific cut-off scores for specific items or scales of the Assessment for Signal Clients for feedback. Rather the existing evidence suggests that delivering the ASC feedback along with the decision tree effects outcome.

According to Kimball (2010), the Cronbach alpha coefficient for each scale is: Therapeutic Alliance (.87); Motivation for Therapy (.81); Social Support (.88); and Life Events (.81). Similarly, Probst et al. (2014b) found similar values in Germany with Cronbach Alphas ranging from .89-.71. These coefficients were based on all clients in treatment (both on-track and off-track clients). Generally, items in the scales contributed to their respective internal consistency. Confirmatory factor analysis suggested the factor structure was consistent with the content of the designed scales (Al-Alim, 2012). More detailed presentation of the theoretical background of the Assessment for Signal Clients scales can be found in the administration and scoring manual (Lambert et al., 2007).

\section{Procedure}

The Assessment for Signal Clients results for not on-track (NOT) clients from the Simon et al. (2012) study $(N=107)$ were analyzed through frequency counts of signal items (items identified as meeting the below $20 \%$ cut-off) to identify the frequency of problematic items and 
their respective scales. Of note, analyses focused on patterns of signal alerts for both the Assessment for Signal Clients items and the overall alliance scale, motivation scale, social support scale and life event scale for two reasons. First, the inclusion of item signal alerts seemed appropriate, given that the OQ-Analyst provides feedback to therapists for both individual items, as well as the alliance, motivation, social support, and life event scales. Due to the manner in which the Assessment for Signal Clients is scored, a client may receive a signal alert on an item but not signal on the scale associated with that item. Thus, important information may have been ignored if the focus of analysis was solely on scales. Second, 45 out of the 107 NOT clients (41.4\%) did not obtain signal alerts for any of the four scales (see Table 1 for patterns of scale signal alerts), thus leaving a narrow range of power and complexity with which to detect patterns in the data.

In addition to frequency counts, a cluster analysis was performed in order to identify similar and distinct problematic participant types. A Cluster analysis is a multivariate procedure for separating cases into their naturally occurring homogeneous groupings. The aim of cluster analysis research "is to uncover structure in data without making prior assumptions" (Chignell \& Stacey, 1981, p. 133). Although there are numerous ways to perform a cluster analysis, there are generally four steps: First, data are collected on a large sample of subjects. Second, profile similarities among subjects are computed using a coefficient such as correlation or Euclidian distance. Third, a computer algorithm is used to search for homogeneous subgroups based on objective criteria. Specifically, subjects in one cluster are deemed to be more similar to each other on objective criteria than they are to subjects classified into another cluster. Fourth, the clusters are typically replicated with new samples and validated against several outcome measures. As a final step in these analyses, the relationship among constructs on the Assessment 
for Signal Clients were examined using log-linear analysis to identify potential co-occurring patterns of endorsement among Assessment for Signal Clients items and scales. Log-linear modeling is a technique that can be used to identify patterns in frequency counts across levels of categorical variables, and provides information for both main effects and interactions between variables (Agresti, 1990).

\section{Results}

Analyses were carried out using IBM SPSS Statistics Version 21. When referring to Assessment for Signal Clients items and scales, Therapeutic Alliance will be abbreviated "TA," Motivation “M," Social Support "SS," and Life Events “LE.”

\section{Descriptive Information}

As shown in Table 1, 41.4\% of the not-on-track clients received signal alerts for none of the Assessment for Signal Clients scales. Clinicians using the Assessment for Signal Clients feedback can expect a large minority of clients not to have enough signaling items within a particular scale to identify a significant overall alarm based on therapeutic alliance, motivation, social support or life event alerts. Forty individuals (37.5\%), another large minority of clients, alerted on a single scale. Importantly, the most common scale to achieve alert status was Social Support (SS; 31 alerts, $28.9 \%$ of clients identified as having significant problems in this area), followed by Therapeutic Alliance (TA) and the Motivation (M) scale (each scale with 19 alerts, $17.7 \%$ of clients), followed by the Life Events (LE) scale (13 alerts, $12.1 \%$ of clients). Overall these data suggest the need for clinicians (and designers of the OQ-Analyst) to be prepared with as many suggestions as possible for interventions related specifically to social support.

Table 2 provides descriptive information regarding signal alert patterns at the item level, and Table 3 provides frequency information for the total number of signal alerts per item across 
the 107 participants. Measures of central tendency reveal that it was common for each patient to have two to four signal alert items within each scale and around 10 item signal alerts total. Table 3 provides the rank ordering for the frequency with which the cut-offs were identifying item endorsement problems that may need to be addressed in psychotherapy with not-on-track cases. Interestingly, therapists received alerts on about $50 \%$ of not-on-track participants on two of the items within the social support scale, the highest percentage for any ASC items. As can be seen in Table 3, the top two Social Support items were quite similar in nature with both suggesting the absence of a person in the client's network of close friends or family who could be trusted with emotionally challenging feelings. 
Table 1

Frequency of Patterns of Signal alerts for Scales of the Assessment for Signal Clients

\begin{tabular}{lc}
\hline \multicolumn{1}{c}{ ASC scale(s) with Signal Alert } & $\begin{array}{c}\text { Total number (percentage) of } \\
\text { participants with signal alerts for } \\
\text { scale(s) }(n=107)\end{array}$ \\
\hline No signal alerts for ASC scales & $45(41.4 \%)$ \\
Social Support (only) & $20(18.69 \%)$ \\
Life Events (only) & $9(8.4 \%)$ \\
Motivation (only) & $6(5.6 \%)$ \\
Therapeutic Alliance (only) & $5(4.6 \%)$ \\
Social Support and Motivation & $4(3.7 \%)$ \\
Therapeutic Alliance, Social Support, and & $4(3.7 \%)$ \\
Motivation & \\
Therapeutic Alliance and Motivation & $4(3.7 \%)$ \\
Therapeutic Alliance and Life Events & $3(2.8 \%)$ \\
Therapeutic Alliance and Social Support & $2(1.8 \%)$ \\
Therapeutic Alliance, Social Support, Motivation, \\
and Life Events
\end{tabular}


Table 2

Descriptive Statistics for Item Signal Alerts

\begin{tabular}{|c|c|c|c|c|c|}
\hline & $\begin{array}{c}\text { All } 40 \text { ASC } \\
\text { items }\end{array}$ & $\begin{array}{c}\text { Therapeutic } \\
\text { Alliance Scale } \\
\text { items }\end{array}$ & $\begin{array}{l}\text { Social Support } \\
\text { Scale items }\end{array}$ & $\begin{array}{l}\text { Motivation } \\
\text { Scale items }\end{array}$ & $\begin{array}{l}\text { Life Events } \\
\text { Scale items }\end{array}$ \\
\hline $\begin{array}{l}\text { Total number of } \\
\text { signal alerts } \\
\text { (across } \\
\text { participants) }\end{array}$ & 1,074 & 296 & 330 & 221 & 227 \\
\hline $\begin{array}{l}\text { Mean number of } \\
\text { signal alerts (per } \\
\text { participant) and } \\
\text { SD }\end{array}$ & $10.03 \pm 6.29$ & $2.77 \pm 2.99$ & $3.08 \pm 2.54$ & $2.06 \pm 2.07$ & $2.12 \pm 1.95$ \\
\hline $\begin{array}{l}\text { Median number of } \\
\text { signal alerts (per } \\
\text { participant) }\end{array}$ & 10 & 2 & 3 & 1 & 2 \\
\hline $\begin{array}{l}\text { Mode number of } \\
\text { signal alerts (per } \\
\text { participant) and } \\
\text { percentage of } \\
\text { participants with } \\
\text { that value }\end{array}$ & $10(9.3 \%)$ & $0(28.9 \%)$ & $0(18.7 \%)$ & $0(28.0 \%)$ & $0(27.1 \%)$ \\
\hline
\end{tabular}


Table 3

Frequency and Item Signal Alerts of Across Not-On-Track Clients

Total Number and

Percentage of Signal Alerts Assessment for Signal Clients Items

for not-on-track Clients

$107-60$ alerts (100 to $56 \%) \quad$ None

59-50 alerts (55 to 47\%) \#16 There was a special person who was around when I was in need. \#17 There was a special person with whom I could share my joys and sorrows.

49-40 alerts (46 to 37\%) \#4 I felt like I could trust my therapist completely.

39-30 alerts (36 to 28\%) \#1 I felt cared for and respected as a person. \#3 I found the suggestions my therapist made were useful. \#9 My therapist seemed to be glad to see me. \#10 My therapist and I seemed to work well together to accomplish what I want. \#12 I could count on my friendships when things went wrong. \#15 I got the emotional help and support I needed from someone in my family. \#22 Some subjects were so sensitive I couldn't talk with anyone about them. \#28 I have no desire to work out my problems. \#32 I had an interaction with another person that I found upsetting. \#33 I felt rejected or betrayed by someone. \#38 I had health problems (such as physical pain).

29-20 alerts (27 to 19\%) \#6 I felt there was a breakdown in the relationship with my therapist. \#7 I felt like my therapist disapproved of me. \#8 At times, the tone of my therapist's voice seemed critical or impatient. \#13 I could talk about problems with my friends. \#14 I could talk about problems with my family. \#20 I felt accepted by someone other than my therapist. \#21 I felt connected to a higher power. \#24 Honestly, I really don`t understand what I can get from therapy. \#25 I am not really sure what to work on in therapy. \#26 I had thoughts about quitting therapy; it's just not for me. \#27 I don`t think therapy will help me feel any better. \#29 Although I am currently unhappy with life, there is nothing I can do about it now. \#31 I am in therapy because someone is requiring it of me. \#34 I made a mistake that I can`t undo. \#35 I received bad news that was difficult for me. \#37 There was trouble at home, work, or school. \#40 I had difficulty adjusting to an occurrence in my life.

\section{Cluster Analysis}

In order to determine the natural groupings among not-on-track patients, a cluster analysis was performed using the 40 Assessment for Signal Clients items. The two-step cluster method (with log-likelihood as the distance measure) was chosen to conduct the analysis because 
of its utility for categorical data. The number of clusters was chosen automatically as part of the SPSS algorithm using Schwarz's Bayesian Criterion and resulted in a three cluster solution. Table 4 provides frequency data for the 40 Assessment for Signal Clients items grouped by cluster.

As can be seen in Table 4, the 21 not-on-track clients in cluster 1 had high rates of signal endorsement of items in the therapeutic alliance and motivation scales. As a result, we labeled this the "Internal" cluster to represent their focus on in-therapy or internal issues that may influence their flagging progress in therapy. In addition, clients in cluster 1 endorsed the largest number of all items in the signal range with an average of $17.9(S D=4.27)$. The 31 clients in cluster 2 had higher signal endorsement rates of items in the life events and social support scales. As a result, we labeled them the "External" cluster to represent their focus on outside of therapy issues as influencing their flagging progress in therapy. Individuals in cluster 2 endorsed an average of 12.5 items $(S D=3.59)$ in the signal range on the Assessment for Signal Clients. The 55 not-on-track clients in Cluster 3 endorsed an average of 5.3 items $(S D=3.16)$ in the signal range and had no particular pattern of item endorsement. No individual item had more than $40 \%$ signal endorsement. The two items endorsed by individuals in cluster 3 at greater than $30 \%$ were a social support item \#16 "There was a special person who was around when I was in need" and a motivation item \#31 "I am in therapy because someone is requiring it of me".

A one-way ANOVA comparing the mean number of items endorsed within each cluster was significant, $F(2,104)=109.80, p<.001$ and post hoc tests (Scheffe) indicated that all three averages were significantly different from each other. 
Table 4

Cluster Item Frequency

\begin{tabular}{|c|c|c|c|c|c|c|}
\hline \multirow[t]{2}{*}{ Item Type } & \multicolumn{2}{|c|}{ Cluster 1} & \multicolumn{2}{|c|}{ Cluster 2} & \multicolumn{2}{|c|}{ Cluster 3} \\
\hline & $\#$ of items & $\%$ of items & \# of items & $\%$ of items & $\#$ of items & $\%$ of items \\
\hline \#1 (TA) & 18 & 85.7 & 7 & 22.6 & 7 & 12.7 \\
\hline \#2 (TA) & 11 & 52.4 & 1 & 3.2 & 0 & 0 \\
\hline \#3 (TA) & 20 & 95.2 & 6 & 19.4 & 5 & 9.1 \\
\hline \#4 (TA) & 17 & 81.0 & 14 & 45.2 & 12 & 21.8 \\
\hline \#5 (TA) & 10 & 47.6 & 6 & 19.4 & 2 & 3.6 \\
\hline \#6 (TA) & 10 & 47.6 & 9 & 29.0 & 4 & 7.3 \\
\hline \#7 (TA) & 12 & 57.1 & 7 & 22.8 & 10 & 18.2 \\
\hline \#8 (TA) & 9 & 42.9 & 4 & 12.9 & 7 & 12.7 \\
\hline \#9 (TA) & 15 & 71.4 & 10 & 32.3 & 10 & 18.2 \\
\hline \#10 (TA) & 20 & 95.2 & 10 & 32.3 & 9 & 16.4 \\
\hline \#11 (TA) & 11 & 52.4 & 3 & 9.7 & 0 & 0 \\
\hline \#12 (SS) & 9 & 42.9 & 21 & 67.7 & 5 & 9.1 \\
\hline \#13 (SS) & 6 & 28.6 & 12 & 38.7 & 3 & 5.5 \\
\hline$\# 14$ (SS) & 2 & 9.5 & 18 & 58.1 & 2 & 3.6 \\
\hline \#15 (SS) & 9 & 42.9 & 20 & 64.5 & 4 & 7.3 \\
\hline \#16 (SS) & 13 & 61.9 & 20 & 64.5 & 21 & 38.2 \\
\hline \#17 (SS) & 12 & 57.1 & 23 & 74.2 & 16 & 29.1 \\
\hline \#18 (SS) & 2 & 9.5 & 7 & 22.6 & 4 & 7.3 \\
\hline \#19 (SS) & 5 & 23.8 & 10 & 32.3 & 3 & 5.5 \\
\hline \#20 (SS) & 6 & 28.6 & 15 & 48.4 & 2 & 3.6 \\
\hline \#21 (SS) & 8 & 38.1 & 11 & 35.5 & 7 & 12.7 \\
\hline \#22 (SS) & 8 & 38.1 & 14 & 45.2 & 12 & 21.8 \\
\hline \#23 (M) & 15 & 71.4 & 3 & 9.7 & 1 & 1.8 \\
\hline \#24 (M) & 17 & 81.0 & 1 & 3.2 & 5 & 9.1 \\
\hline \#25 (M) & 13 & 61.9 & 8 & 25.8 & 7 & 12.7 \\
\hline \#26 (M) & 14 & 66.7 & 1 & 3.2 & 11 & 20.0 \\
\hline \#27 (M) & 13 & 61.9 & 4 & 12.9 & 5 & 9.1 \\
\hline \#28 (M) & 9 & 42.9 & 9 & 29.0 & 14 & 25.5 \\
\hline \#29 (M) & 6 & 28.6 & 10 & 32.3 & 8 & 14.5 \\
\hline \#30 (M) & 6 & 28.6 & 2 & 6.5 & 10 & 18.2 \\
\hline \#31 (M) & 8 & 38.1 & 4 & 12.9 & 17 & 30.9 \\
\hline \#32 (LE) & 5 & 23.8 & 18 & 58.1 & 14 & 25.5 \\
\hline \#33 (LE) & 6 & 28.6 & 18 & 58.1 & 8 & 14.5 \\
\hline \#34 (LE) & 6 & 28.6 & 18 & 58.1 & 8 & 14.5 \\
\hline \#35 (LE) & 7 & 33.3 & 7 & 22.6 & 9 & 16.4 \\
\hline \#36 (LE) & 2 & 9.5 & 6 & 19.4 & 3 & 5.5 \\
\hline \#37 (LE) & 5 & 23.8 & 12 & 38.7 & 12 & 21.8 \\
\hline \#38 (LE) & 2 & 9.5 & 17 & 54.8 & 12 & 21.8 \\
\hline \#39 (LE) & 6 & 28.6 & 8 & 25.8 & 2 & 3.6 \\
\hline$\# 40$ (LE) & 9 & 42.9 & 10 & 32.3 & 7 & 12.7 \\
\hline
\end{tabular}

Note. TA = Therapeutic Alliance; $S S=$ Social Support; $M=$ Motivation; $L E=$ Life Events.

$N($ Cluster 1$)=21 ; \mathrm{N}($ Cluster 2$)=31 ; \mathrm{N}($ Cluster 3$)=55$. Item percentages were greater than $50 \%$ signal endorsement bolded. 


\section{Loglinear Modeling: Examination of Associations between Variables}

In order to transform the data into categorical form, for the log-linear portion of the analysis, not-on-track participants were categorized based on the number of item signal alerts a participant received on items within each separate scale. Four dummy variables were created (i.e., Therapeutic Alliance (TAd), Social Support (SSd), Motivation (Md), Life Events (LEd)) and a participant was assigned a " 0 " value for a scale if he or she was identified as having 0 or 1 item alert(s) within a scale and a " 1 " value if he or she was identified as having 2 or more item alerts within a scale. For example, if a not-on-track participant had 3 item alerts (out of the 11 possible items) within the TA scale, he or she was coded as a "1" for TAd and would be considered a "high scorer" for that scale. If he or she had 0 item alerts within the SS scale (out of the 11 possible items), he or she was coded as a " 0 " for SSd and would be considered a "low scorer" for that scale. Our cut-off criteria for categorization as a "high" or "low scorer" was to choose the cut-off that resulted in the most evenly distributed cell frequency counts within each dummy variable (e.g., $50 \%$ of participants were valued " 0 " for $\mathrm{SSd}$ ), which is commonly recommended as a means to meet the minimum expected cell frequencies for goodness-of-fit tests (Warner, 2008, p. 156).

As shown in Table 5 (contingency table for the four dummy variables), for the current analysis, $43 \%$ of the expected cell frequencies were below five, which is less than recommended for cell frequency analyses (Wickens, 1989). This could be considered a limitation of the analysis, though none of the frequencies were less than 1 (the lowest frequency was 2.5), and there have been arguments that this condition is likely acceptable (Everitt, 1977). Thus, though the results might be interpreted with this limitation in mind, this issue was deemed minor enough 
that analysis could proceed. A correction for continuity was applied for goodness-of-fit tests, such that 0.5 was added to every negative observed and expected cell frequency and subtracted from every positive cell frequency (Wickens, 1989).

A backward elimination approach was taken for the current study, which means that a model was first developed so as to include all main effects and higher order associations (i.e., terms) and then, starting with the highest order interaction (i.e., TAd x SSd x Md x LEd), a term was removed one by one, and a statistical test was performed to determine whether dropping the term lead to a significant change in the model. For a more in-depth explanation of log-linear analysis, please see Agresti (1990) and Wickens (1989). Removal of 4- and 3-way associations did not result in significant changes in the model. The following 2-way interactions were found significant at $p<0.05: \operatorname{SSd} \times \operatorname{Md}(p=0.042), \operatorname{SSd} x \operatorname{LEd}(p=0.04)$, and TAd $\mathrm{x} \operatorname{Md}(p=0.00)$. Both Pearson (chi-square $=4.224, d f=7, \mathrm{p}=0.754)$ and Likelihood Ratio $($ chi-square $=4.446, d f$ $=7, \mathrm{p}=0.727$ ) goodness-of-fit tests were not significant, indicating a good fit to the model. In further support for model fit, none of the standardized residuals for the final model were greater than \pm 1.96 .

Table 6 includes parameter estimates, z-scores, significance levels, and confidence intervals for each main effect and interaction estimates of parameters are calculated as odds expressed in logarithmic terms. Significant effects occurred solely for the TAd x Md interaction (estimate $=0.38, z=3.314, p=0.001,95 \%$ confidence interval: 0.155 to 0.602 ) and SSd main effect (estimate $=-0.32, z=-2.182, p=0.005,95 \%$ confidence interval: -0.546 to -0.098 ). These results suggest that if a participant was a low scorer for TA, they were also likely (above chance) to be a low scorer on Md, and that was generally less likely (below chance) for participants to be low scorers on SSd. This is consistent with the preceding descriptive tables 
and cluster analysis, which showed that a relatively higher percentage of not-on-track

participants received signal alerts for the SS items and scale, and there seems to be a grouping of clients who align on "internal" to therapy issues. The lack of significant effects for the other two interactions indicated for the model (SSd x Md and SSd x LEd) suggest less confidence can be held for their association, but this potential relationship might be a consideration for future studies.

Table 5

Contingency table for Dummy Variables

\begin{tabular}{|c|c|c|c|c|c|}
\hline & & \multicolumn{2}{|c|}{$S S d=0$} & \multicolumn{2}{|c|}{$S S d=1$} \\
\hline & & $L E d=0$ & $L E d=1$ & $L E d=0$ & $L E d=1$ \\
\hline \multirow[t]{2}{*}{$T A d=0$} & $M d=0$ & $\begin{array}{c}13 \\
\text { (Low all } \\
\text { scales) }\end{array}$ & $\begin{array}{c}6 \\
\text { (LEd High) }\end{array}$ & $\begin{array}{c}8 \\
\text { (SSd high) }\end{array}$ & $\begin{array}{c}14 \\
\text { (LEd and SSd } \\
\text { high) }\end{array}$ \\
\hline & $M d=1$ & $\begin{array}{c}3 \\
\text { (Md high) }\end{array}$ & $\begin{array}{c}2 \\
\text { (LEd and Md } \\
\text { high) }\end{array}$ & $\begin{array}{c}7 \\
\text { (SSd and Md } \\
\text { high) }\end{array}$ & $\begin{array}{c}4 \\
\text { (Md, SSd, and } \\
\text { LEd high) }\end{array}$ \\
\hline \multirow[t]{2}{*}{$T A d=1$} & $M d=0$ & $\begin{array}{c}3 \\
\text { TAd high }\end{array}$ & $\begin{array}{c}2 \\
\text { (TAd and LEd } \\
\text { high) }\end{array}$ & $\begin{array}{c}3 \\
\text { (TAd and SSd } \\
\text { high) }\end{array}$ & $\begin{array}{c}6 \\
\text { TAd, SSd, and } \\
\text { LEd high) }\end{array}$ \\
\hline & $M d=1$ & $\begin{array}{c}2 \\
\text { (TAd and Md } \\
\text { high) }\end{array}$ & $\begin{array}{c}5 \\
\text { (TAd, Md, } \\
\text { and LEd high) }\end{array}$ & $\begin{array}{c}7 \\
\text { (TAd, Md, and } \\
\text { SSd high) }\end{array}$ & $\begin{array}{c}22 \\
\text { (TAd, Md, SSd } \\
\text { and LEd high) }\end{array}$ \\
\hline
\end{tabular}

Note. High = high scorer. SSd = Social Support dummy variable; LEd = Life Events dummy variable; $\mathrm{TAd}=$ Therapeutic Alliance dummy variable; $\mathrm{Md}=$ Motivation dummy variable . 
Table 6

Parameter Estimates for Main Effects and Interactions of Dummy Variables

\begin{tabular}{|c|c|c|c|c|}
\hline Effect & $\begin{array}{c}\text { Parameter } \\
\text { Estimate }\end{array}$ & z-score & Significance & $\begin{array}{c}\text { Confidence Interval } \\
(95 \%)\end{array}$ \\
\hline TAd x SSd x Md x LEd & 0.05 & 0.43 & 0.66 & -0.17 to 0.27 \\
\hline TAd $\times$ SSd $\times$ Md & -0.03 & -0.29 & 0.77 & -0.26 to 0.19 \\
\hline TAd $x$ SSd $x$ LEd & -0.01 & -0.09 & 0.92 & -0.23 to 0.21 \\
\hline TAd $\mathrm{x}$ Md $\mathrm{x}$ LEd & -0.14 & -1.23 & 0.22 & -0.36 to 0.08 \\
\hline SSd $x$ Md $x$ LEd & 0.13 & 1.14 & 0.25 & -0.09 to 0.35 \\
\hline TAd $\times$ SSd & 0.11 & 0.97 & 0.33 & -0.11 to 0.33 \\
\hline TAd $x$ Md & 0.38 & 3.31 & $0.001 *$ & 0.15 to 0.60 \\
\hline $\mathrm{SSd} \times \mathrm{Md}$ & 0.16 & 1.40 & 0.16 & -0.06 to 0.38 \\
\hline TAd $x$ LEd & 0.20 & 1.76 & 0.08 & -0.02 to 0.42 \\
\hline SSd x LEd & 0.15 & 1.28 & 0.19 & -0.08 to 0.37 \\
\hline Md x LEd & 0.06 & 0.52 & 0.60 & -0.16 to 0.28 \\
\hline TAd & 0.12 & 1.06 & 0.29 & -0.10 to 0.34 \\
\hline $\mathrm{SSd}$ & -0.32 & -2.82 & $0.005^{*}$ & -0.55 to -0.098 \\
\hline $\mathrm{Md}$ & 0.07 & 0.63 & 0.53 & -0.15 to 0.29 \\
\hline LEd & -0.07 & -0.62 & 0.54 & -0.29 to 0.15 \\
\hline
\end{tabular}

Note. ${ }^{*} p<0.01$

\section{Discussion}

Lambert and colleagues found that alerting therapists to probable treatment failure improved psychotherapy outcomes for not-on-track clients but still a portion went on to deteriorate or were not improved at termination. In an attempt to further prevent treatment failure they developed a problem-solving tool based on a 40-item self-report scale (the Assessment for Signal Clients; ASC) administered if and when a client became not-on-track for a positive 
outcome. Use of this intervention in controlled trials further reduced deterioration and bolstered positive treatment response (Shimokawa et al., 2010). Southwick and Lambert (2014) found in a qualitative study that therapists tend to use the Clinical Support Tools in a manner consistent with their design.

The current study explored Assessment for Signal Clients data from 107 predicted treatment failures from a hospital-based outpatient clinic (Simon et al., 2012) in order to describe the frequency and nature of problems clients noted on the Assessment for Signal Clients that met criterion (Lambert et al., 2007) for being problematic. Both scale feedback and specific item feedback were examined in the current study. Within the current sample of outpatients who were predicted to deteriorate about $58 \%$ of patients had enough problems within a unique scale to trigger a scale alarm signal. Surprisingly, the Social Support scale (instead of the Therapeutic Alliance scale) was triggered most often, with $29 \%$ of all not-on-track clients meeting criterion for an overall problem in this area. About $40 \%$ of clients did not have enough items within any of the four scales to bring the overall scale score to the critical threshold for an alarm. At the item level clients had an average of 10 items $(S D=6.29)$ that passed the threshold for an alert, giving therapists numerous specific issues that they could explore with the not-on-track client while also limiting the list of likely problems.

As would be expected from the scale results, the most frequently identified problematic items were from the Social Support scale ("There was a special person who was around when I was in need." "There was a special person with whom I could share my joys and sorrows." "I could count on my friendships when things went wrong."). Cumulatively, these items suggest that many not-on-track clients are missing a close person in their social network with whom they can confide at the time they go off-track for a positive outcome. This suggests the need for 
therapists to consider interventions that could strengthen the social network of such clients, help them with social skills, including a significant other in the psychotherapy process, or make referrals to group psychotherapy or support groups as an adjunct to individual psychotherapy. These results are consistent with the findings of Probst et al. (2014a) who suggested that social support item were so commonly endorsed that the decision tree that guides problem solving for therapists should be reorganized so that social support precedes rather than follows alliance. Such a change in emphasis may come as a relief to therapists as it implies that predicted deterioration is most likely caused by a weak social support network rather than themselves. Of course replication of these results will be necessary before modifying the decision tree.

The Assessment for Signal Clients also identified alliance problems in not-on-track cases with items like: "I felt like I could trust my therapist completely" and "My therapist seemed glad to see me." There also seemed to be evidence of collaboration difficulties leading the way (e.g., "My therapist and I seemed to work well together to accomplish what I want."). Certainly alliance problems would seem to be more easily solved than social support difficulties since the therapist can rather readily become more responsive to patient needs than engineer their social environment. Therapists may also find some relief in knowing that bond problems (e.g., empathy, respect) do not fully explain the off-track client's negative change. It may be a problem of renegotiating the therapeutic contract by (re)presenting the therapeutic rationale and its connection to in-session therapeutic procedures. This consideration also applies to motivational problems. Not-on-track clients require help in overcoming hindering motivational states. Recall the association between the alliance and motivational items as shown in the results of loglinear modeling and the identification of an "internal" cluster in the cluster analysis. Hansen, Lambert and Vlass (2014) in their study of off- track clients compared to early dramatic treatment 
responders found little difference in the bond aspects of the alliance, but evidence of task aspects of the alliance and motivational problems.

When considering clusters or groups of not-on-track clients with similar profiles, three clusters emerged. Cluster 1 included about $20 \%$ of the not-on-track clients who endorsed a larger number of items in the signal range (an average of 17), with a pattern of high endorsement in the therapeutic alliance and motivation scales. If this pattern holds in future studies, clinicians might expect about $20 \%$ of clients who are not on track to report a variety of problems "internal" to therapy (i.e., alliance and motivation to change). In contrast, cluster 2 included about $29 \%$ of the not-on-track clients who had an average number of items endorsed (12) but a different pattern in that they endorsed more social support and life event items (i.e. external to therapy issues) as contributing to their lack of progress in treatment.

These two clusters may represent contrasting explanations for treatment deterioration. The internal cluster may relate to a breakdown in the process of therapy itself. A therapist interpreting the results of an Assessment for Signal Clients administration may focus cluster 1 clients on factors that relate directly to the process of therapy and may also choose to adjust interventions accordingly. However, cluster 2 explains deterioration in terms of problems external to therapy and suggests a client having a context or experiences in that context that exceed her/his resources for coping. There may be merit for future discussion on an internal versus external split in explaining treatment deterioration.

The remaining $51 \%$ of clients, in cluster 3 , had no specific pattern of item endorsement on the Assessment for Signal Clients, although two facts stand out. First, 30\% of the clients in cluster 3 report participating in treatment as a result of pressure from a third party and $38 \%$ reported problems with social support. Second, not-on-track clients in cluster 3 had an overall 
lower rate of endorsement of Assessment for Signal Clients items in general. This may suggest that something other than alliance, motivation, social support, or life events was contributing to their poor progress in therapy.

Alliance and motivation problems appear to be related as "internal to therapy" problems and indicate to a therapist working with such problems to use interventions within the context of therapy that focus on improving the relationship and enhancing motivation. External to therapy issues (i.e., social support and life events) may present more of a challenge, since therapists have a less direct influence on such issues. Therapists working with such clients may need to more seriously consider a client's context outside of therapy and how relationships with others may contribute to distress. It is noted, however, that interventions from a broad spectrum of theoretical orientations may be used to enhance social support. Behavioral activation may include taking action to enhance social support while cognitive restructuring may help a client reframe their perspective on available sources of support. Humanistic and psychodynamic approaches may focus on a client's relational framework and on enhancing relationships to maximize a client's benefit from these relationships.

More research may be needed to address how therapists can enact social support interventions. Policy-makers may take note how this study's findings emphasize that possible explanations for treatment deterioration for mental health clients tend to be complex and idiosyncratic. Not-on-track clients may require the commitment of more clinical resources to determine how best to provide effective intervention.

The cluster analysis provides an interesting starting point for further examination of noton-track clients. In this sample, three distinct profile types emerged that provide provocative clinical information that may be useful to the therapist. Of particular help is the finding that $50 \%$ 
of the not-on-track clients may have either alliance and motivation issues - internal cluster - or social support and disruptive life events - external cluster. The remaining $50 \%$ it is a bit more difficult to identify a prototypical pattern. Given the lack of data examining cluster stability and reliability, these initial findings must remain tentative. More cluster analyses may be conducted with similar, as well as different populations (e.g., not-on-track clients in different settings) to determine whether these findings can be replicated. Future studies may further clarify the types and groups of clients who are not on track in treatment. In particular, future studies may address how these clusters relate to eventual outcome, including recovery or deterioration. While these results appear to be valid for a general mental health population (mood and anxiety disorders), these clusters may also be studied relative to diagnosis or other presenting problems. Should these results be replicated and found to generalize across other treatment populations, typing patients and providing this information to therapists could speed problem solving with at least a portion of not-on-track patients. 


\section{References}

Agresti, A. (1990). Categorical data analysis. New York: Wiley.

Al-Alim, O. (2012). Psychometric evaluation of a Dutch translation of the Assessment for Signal Clients questionnaire (Unpublished doctoral dissertation). Leiden University, Leiden, Netherlands.

Bohart, A. C. \& Wade, A. G. (2013). The client in psychotherapy. In Michael J. Lambert (Ed.), Bergin and Garfield's handbook of psychotherapy and behavior change (6 ${ }^{\text {th }}$ ed., pp. 219257). New York, NY: Wiley.

Chignell, M. H., \& Stacey, B. G. (1981). The classification of patients into diagnostic groups using cluster analysis. Journal of Clinical Psychology, 37(1), 151-153.

Chronister, J. A., Johnson, E. K., \& Berven, N. L. (2006). Measuring social support in rehabilitation. Disability \& Rehabilitation, 28(2), 75-84.

doi:10.1080/09638280500163695

Ellsworth, J. R., Lambert, M. J., \& Johnson, J. (2006). A comparison of the Outcome Questionnaire-45 and Outcome Questionnaire-30 in classification and prediction of treatment outcome. Clinical Psychology \& Psychotherapy, 13(6), 380-391. doi:10.1002/cpp.503

Everitt, B. (1977). The analysis of contingency tables. London: Chapman and Hall.

Finch, A. E., Lambert, M. J., \& Schaalje, B. G. (2001). Psychotherapy quality control: The statistical generation of expected recovery curves for integration into an early warning system. Clinical Psychology \& Psychotherapy, 8(4), 231-242. doi:10.1002/cpp.28

Hannan, C., Lambert, M. J., Harmon, C., Nielsen, S. L., Smart, D. M., Shimokawa, K., \& Sutton, 
S. W. (2005). A lab test and algorithms for identifying patients at risk for treatment failure. Journal of Clinical Psychology: In Session, 61(2), 155-163.

Hansen, B., Lambert, M. J., \& Vlass, E. N. (2014). Sudden gains: A pluralistic approach to the patient and therapist experience. (Unpublished doctoral dissertation). Brigham Young University, Provo, UT.

Horvath, A. O., Del Re, A. C., Flückiger, C., \& Symonds, D. (2011). Alliance in individual psychotherapy. Psychotherapy, 48(1), 9-16. doi:10.1037/a0022186

Kimball, K.L. (2010). Toward determining best items for identifying therapeutic problem areas. (Unpublished doctoral dissertation). Brigham Young University, Provo, UT.

Lambert, M. J., Bailey, R., Kimball, K., Shimokawa, K., Harmon, S. C., \& Slade, K. (2007). Clinical support tool manual-brief version-40. Salt Lake City: OQ Measures.

Lambert, M. J., \& Cattani-Thompson, K. (1996). Current findings regarding the effectiveness of counseling: Implications for practice. Journal of Counseling \& Development, 74(6), 601608.

Lambert, M. J., Kahler M., Harmon C., Burlingame G. M., Shimokawa K., \& White M., (2013). Outcome questionnaire OQ-45.2. (4 $4^{\text {th }} E d$.). SLC: OQ Measures, LLC.

Lambert, M. J., Whipple, J. L., Bishop, M. J., Vermeersch, D. A., Gray, G. V., \& Finch, A. E. (2002). Comparison of empirically-derived and rationally-derived methods for identifying patients at risk for treatment failure. Clinical Psychology \& Psychotherapy, 9(3), 149-164. doi:10.1002/cpp.333

Lindfors, O., Ojanen, S., Jääskeläinen, T., \& Knekt, P. (2014). Social support as a predictor of the outcome of depressive and anxiety disorder in short-term and long-term 
psychotherapy. Psychiatry Research, 216(1), 44-51.

doi:10.1016/j.psychres.2013.12.050

Probst, T., Lambert, M. J., Dahlbender, R. W., Loew, T. H., Thomas H., \& Tritt, K. (2014).

Providing patient progress feedback and clinical support tools to therapists: Is the therapeutic process of patients on-track to recovery enhanced in psychosomatic in-patient therapy under the conditions of routine practice? Journal of Psychosomatic Research, 76(6), 477-484. doi:10.1016/j.jpsychores.2014.03.010

Probst, T., Lambert, M. J., Loew, T. H., Dahlbender, R. W., \& Tritt, K. (2015). Extreme deviations from expected recovery curves and their associations with therapeutic alliance, social support, motivation, and life events in psychosomatic in-patient therapy. Psychotherapy Research, 25(6), 714-723. http://dx.doi.org/10.1080/10503307.2014.981682

Prochaska, J. O. \& Norcross, J. C. (2003). Systems of psychotherapy: A transtheoretical analysis ( $5^{\text {th }}$ ed.). Pacific Grove, Ca: Brooks/Cole.

Safran, J. D., \& Muran, J. C. (2000). Resolving therapeutic alliance ruptures: Diversity and integration. Journal of Clinical Psychology, 56(2), 233-243.

Shimokawa, K., Lambert, M. J., \& Smart, D. W. (2010). Enhancing treatment outcome of patients at risk of treatment failure: Meta-analytic and mega-analytic review of a psychotherapy quality assurance system. Journal of Consulting and Clinical Psychology, 78, 298-311.

Simon, W., Lambert, M. J., Harris, M. W., Busath, G., \& Vazquez, A. (2012). Providing patient progress information and clinical support tools to therapists: Effects on patients at risk for treatment failure. Psychotherapy Research, 22, 638-647. 
Spielmans, G. I., Masters, K. S., \& Lambert, M. J. (2006). A comparison of rational versus empirical methods in the prediction of psychotherapy outcome. Clinical Psychology \& Psychotherapy, 13(3), 202-214. doi:10.1002/cpp.491

Southwick, J. \& Lambert, M. J. (2014). Therapists experience with structured feedback procedures: Review and discussion of research literature. Unpublished manuscript, Department of Psychology, Brigham Young University, Provo, UT.

Thoits, P. A. (1986). Social support as coping assistance. Journal of Consulting and Clinical Psychology, 54(4), 416.

Vermeersch, D. A., Lambert, M. J., \& Burlingame, G. M. (2000). Outcome Questionnaire: Item sensitivity to change. Journal of Personality Assessment, 74(2), 242-261.

Vermeersch, D. A., Whipple, J. L., Lambert, M. J., Hawkins, E. J., Burchfield, C. M., \& Okiishi, J. C. (2004). Outcome questionnaire: Is it sensitive to changes in counseling center clients? Journal of Counseling Psychology, 51(1), 38-49.

Warner, R.M. (2008). Applied statistics. Los Angeles, London, New Delhi, Singapore: Sage Publications.

Wickens, T. D. (1989). Multiway contingency tables analysis for the social sciences. Hillsdale, NJ: Lawrence Erlbaum Associates, Inc.

Zimet, G. D., Dahlem, N. W., Zimet, S. G., \& Farley, G. K. (1988). The multidimensional scale of perceived social support. Journal of Personality Assessment, 52, 30-41. 